\title{
Pasado, presente y futuro de la Ultrasonografía Obstétrica
}

\author{
Dr. Fernando Sánchez Torres *
}

\section{Nota Bene}

Es válido advertir desde un principio que el autor del presente artículo no es un ultrasonografista o ecografista. Es apenas uno de los miles de ginecobstetras que en el mundo nos servimos de tan admirable e invaluable procedimiento físico no invasivo, que vino a transformar el ejercicio de la profesión médica en muchas de sus especialidades.

No hay duda que la ultrasonografía debe ser tenida como "la gran revolucionaria del quehacer obstétrico" Creo, por eso, que es necesario que los que la usufructuamos conozcamos algo de su biografía y mucho de sus alcances y posibilidades.

\section{La Exploración Física del Cuerpo Humano. La Imagenología}

Desde que la medicina se convirtió en un oficio o profesión, sus cultores anhelaron poder conocer lo que ocurría en el interior del organismo de su paciente enfermo. Producto de esa curiosidad, como también de su ingenio, han sido los distintos medios que ha puesto en uso para conseguirlo. A continuación mencionaré, muy a la carrera, aquellos considerados como verdaderos hitos de la exploración física del cuerpo humano.

En 1819 el médico francés Renato Teófilo Laënnec publica su famoso libro Tratado de la auscultación mediata, en el cual dio a conocer el valor de la exploración auditiva del tórax mediante una cornetilla, procedimiento inventado por él.

Profesor Titular, Emérito y Honorario de la Universidad Nacional de Colombia. Miembro de la Sociedad Colombiana de Historia de la Medicina.
Por su parte, el alemán Hermann Ludwig von Helmholtz presenta en 1850 un aparato que diseñara y contruyera para mirar directamente lo que ocurre en el interior del ojo humano vivo. Por supuesto que ya antes se habían hecho intentos para lograrlo: Johannes Evangelista Purkinje en 1823 y William Cumming en 1846.

El físico alemán Guillermo Conrado Roentgen, experimentando en su laboratorio el efecto de las descargas eléctricas a través de una ampolla o tubo de Crookes, pudo ver sobre un papel la sombra de los huesos de sus manos. Así descubrió lo que él mismo llamó "rayos X", que vendrían a constituirse durante muchos años en el único y más extraordinario método de diagnóstico en medicina, desalojado parcialmente en la actualidad por otro método revolucionario: la ultrasonografía, de la que hablaré más adelante.

Uno de los inconvenientes anotados a los rayos $\mathrm{X}$ es que las imágenes que proporciona son unidimensionales, un tanto difusas a causa de la sobreposición de los tejidos. Se debe al ingeniero electrónico Godfrey Newbold Hounsfield haber adicionado en 1972 los sistematizadores a los rayos $\mathrm{X}$, logrando con ello una definición anatómica detallada, tridimensional. Esta, la Tomografía Axial Computadorizada (TAC) o escanografía, le valió a Hounsfield la adjudicación del Premio Nobel de Medicina en 1979.

La imagenología en medicina pronto se enriqueció con el advenimiento de la Resonancia Nuclear Magnética (RNM), basada en el movimiento de los protones de hidrógeno de los líquidos del cuerpo, movimiento que se hace en una misma dirección cuando los protones se encuentran dentro de un campo magnético. Los núcleos de hidrógeno, estimulados con pulsos de radiofrecuencia, giran sobre su eje. Al suspender el estímulo los protones regresan a su posición y emiten una señal de radiofrecuencia, la cual, detectada por una antena, 
se transmite al computador y este analiza la información y reconstruye las imágenes. Existe consenso en que la RNM tiene su mejor aplicación en ciertos procesos patológicos del sistema nervioso central (33). No obstante, se ha intentado encontrarle utilidad en el terreno obstétrico. En 1983 la empleó el doctor F.W. Smith, de la Universidad de Aberdeen, Escocia, durante el primer trimestre del embarazo en pacientes que iban a ser sometidas a aborto electivo (43).

No sólo por razones técnicas (mala definición por la presencia del líquido amniótico y por la movilidad fetal), sino también por consideraciones éticas, no se ha difundido su empleo en las primeras doce semanas del embarazo, aceptándose que en esta época el ultrasonido es más beneficioso (42), con excepción de aquellos casos de enfermedad trofoblástica gestacional, pues además de que permite su diagnóstico suministra información sobre el grado de invasión miometrial y las características vasculares del tejido (31). En la gestación avanzada es muy útil para el diagnóstico y estudio de la morfología fetal, particularmente en el caso de malformaciones mayores y menores (42). Pese a que no se ha encontrado evidencia de que la RNM ocasione alteraciones cromosómicas, no se descarta la posibilidad de potenciales efectos carcinogenéticos (44).

El descubrimiento de la RNM, tenido como "el más excitante de la medicina diagnóstica", fue dado a conocer por dos grupos distintos de investigadores: uno, encabezado por Félix Bloch de la Universidad de Stanford, (4) y otro, dirigido por Edward Purcell de la Universidad de Harvard (32). Por este hallazgo recibieron el Premio Nobel de Física en 1952. Gracias a ese aporte, el estudio de las propiedades de la materia a nivel molecular pudo ser posible.

\section{Pasado de los Ultrasonidos}

Los antecedentes del revolucionario método ultrasónico se remontan a 1880, cuando los físicos franceses Pierre y Paul Jacques Curie descubrieron el efecto piezoeléctrico, es decir, la producción de una carga eléctrica en respuesta a la aplicación de una presión mecánica sobre algunos materiales, como el cuarzo. Contrariamente, si en esos mismos materiales se aplica voltaje se producen deformaciones mecánicas. El impacto de las ondas sónicas que produce la deformación mecánica puede ser transformado en energía eléctrica y registrado con instrumentos apropiados (16, p. 2).

Se debe al físico francés Paul Langevin haber intentado darle un empleo práctico a ese fenómeno. Por encargo del Gobierno de su país, Langevin usó el sonido de alta frecuencia en la detección de submarinos durante la Primera Guerra Mundial. Años después las ondas ultrasónicas, es decir, aquellas que por su elevado número de ciclos no son perceptibles por el oído humano, comenzaron a ser utilizadas en la industria, especialmente para descubrir fisuras o grietas internas en distintos materiales, gracias a la iniciativa del físico soviético S.Y.Sokolov en 1928. En la década de los 30 la energía ultrasónica se introdujo al campo de la medicina, irradiando con ella el cuerpo, con la esperanza de obtener efectos tonificantes, y aún restauradores $(16$, p. 3$)$.

Terminada la Segunda Guerra Mundial, grupos de investigadores japoneses se interesaron por darles a los ultrasonidos un empleo diagnóstico en medicina. Así fue como en Tokio, el físico Rokuro Uchida construyó en 1949 el primer equipo ultrasónico para esos fines (16, p. 4). Pronto el médico Kenji Tanaka, también de Tokio, creyó encontrar utilidad diagnóstica en lesiones intracraneales. En efecto, en 1952 comunicó que había detectado hematomas y tumores cerebrales. Más luego su aplicación diagnóstica se amplió, explorándose distintas regiones del cuerpo en busca de tumores. Shigeo Satomura y Yasuharu Nimura, de la Universidad de Osaka, introdujeron el Doppler ultrasónico en la investigación cardiovascular (Fig. 1). En 1955 propusieron emplearlo como método para estudiar el movimiento físico en organismos vivos (16, p. 4). Recordemos que el llamado fenómeno o efecto Doppler consiste en la variación de frecuencia de una onda sonora o electromagnética cuando el origen de esa onda y el observador están en movimiento uno respecto al otro. Dicho fenómeno fue descubierto en 1842 por el físico austriaco Christian Doppler. Asociado a la sonografía,

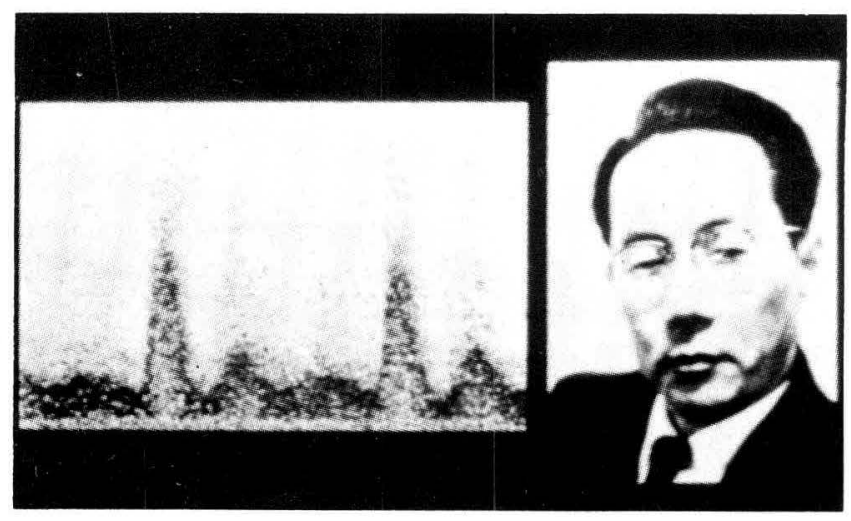

FIGURA 1

A la izquierda, primera imagen espectral de onda-continua de la arteria braquial obtenida en 1964 mediante el efecto Doppler por por el doctor Donald Baker, EE. UU. A la derecha, retrato de Shigeo Satomura, uno de los pioneros de la exploración diagnóstica mediante el efecto Doppler asociado a la transmisión ultrasónica. (Ilustración tomada de "Medical Diagnostic Ultrasound: A Retrospective on Its 40 th Anniversary" publicación de Eastman Kodak). 
el efecto Doppler permite registrar las ondas sonoras que produce el movimiento de la sangre dentro de las arterias y las venas.

Por los datos anteriores pueden advertirse que fueron investigadores japoneses quienes introdujeron de primeros la exploración ultrasónica en medicina. Ellos mismos consiguieron diseñar distintos modelos de aparatos y, de paso, comercializarlos.

Pero conozcamos cómo llegó el diagnóstico ultrasónico al terreno obstétrico. Se debe al profesor Ian Donald, de la Universidad de Glasgow, Escocia, haberlo hecho (Fig. 2). Por desconocer si los ultrasonidos podían tener efectos tóxicos sobre la madre o el feto, comenzó, en 1956, a utilizarlos en ginecología. Los primeros patrones ecográficos diferenciales entre los quistes de ovario y los fibromas del útero se deben a él. Con la colaboración del físico Tom Duggan y del ingeniero Tom Brown, fue afinando las técnicas de exploración. Así el artificio de explorar el abdomen con la vejiga llena o el concurso de un dispositivo para registrar con precisión los diámetros de los objetos examinados, particularmente el diámetro biparietal del feto (16, p. 24).

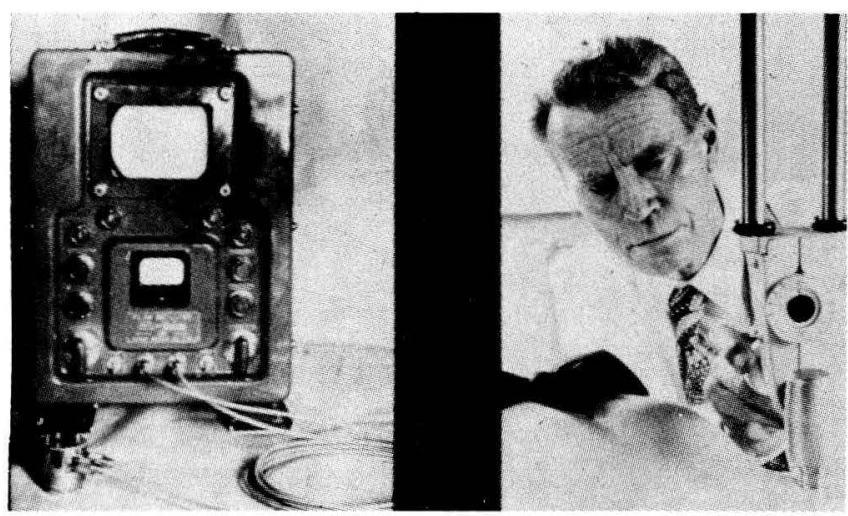

FIGURA 2

A la izquierda, primera versión comercial del equipo ultrasónico desarrollado por el físico Rokuro Uchida en la década de los 40. A la derecha, retrato del doctor Ian Donald, pionero de la ecografía obstétrica. (Ilustraciones tomadas de "Medical Diagnostic Ultrasound: A Retrospective on Its 40 th Anniversary", publicación de Eastman Kodak).

Tras los éxitos de Donald y sus colaboradores, las posibilidades de emplear los ultrasonidos en obstetricia fueron comprobándose en otros países. Horace Thompson y Kenneth Gettesfeld, obstetras del Centro Médico de la Universidad de Colorado, iniciaron su aplicación en los Estados Unidos de Norteamérica. A ellos se debe, entre otras muchas, la descripción de los patrones ecográficos de la placenta, del embarazo múltiple y del crecimiento anormal del feto $(16$, p. 26).

\section{Sitio e Importancia de los Ultrasonidos en el Campo Obstétrico. Daño Potencial}

La sonografía puso fuera de circulación en la actividad obstétrica muchos procedimientos clínicos y de laboratorio que se usaban antes de ella con fines diagnósticos. Por ejemplo, anteriormente era responsabilidad del obstetra obtener mediante recursos clínicos un diagnóstico cierto de embarazo, como también su localización (tópico o ectópico); asimismo, el embarazo múltiple, la edad aproximada de la gestación, la vitalidad del producto y su presentación. Por su parte, de los rayos $\mathrm{X}$ se esperaba que dijeran la última palabra en relación con el número de fetos, con posibles malformaciones, con la inserción segmentaria de la placenta o con la degeneración hidrópica de las vellosidades coriales.

Si bien es cierto que en la actualidad la sonografía ha simplificado el papel del médico al facilitarle la puesta en evidencia de lo que antes era obligación suya descubrir, las nuevas promociones de especialistas en ginecobstetricia han visto mermadas con ella su capacidad inquisidora. El especialista de hoy espera que el aparato y su intérprete, el ecografista, les informen todo lo que está ocurriendo en la intimidad del ambiente endouterino, sin él esforzar su imaginación o poner sus sentidos al servicio de la clínica. Esta sustitución suya por parte de la tecnología ha conducido a que el ginecobstetra haya delegado en el ecografista buena parte del papel que le corresponde desempeñar como responsable del manejo de sus pacientes.

La excesiva confianza que de la sonografía se tiene, ha llevado a que su uso llegue a los límites de la vulgarización, ocasionando con ello su desprestigio y una mala educación por parte del médico. En la práctica se ha establecido una situación ambigua, que no favorece mucho el real alcance y utilidad de los ultrasonidos. Por una parte existe el ginecobstetra práctico que cuenta con la información clínica y que dispone en su consultorio privado de un aparato de ultrasonidos, sin tener la suficiente preparación técnica para darle un empleo adecuado. Esto, que puede considerarse como "mala práctica", se presta para comprometer gravemente a quien hace de ecografista, pues de seguro muchas veces dejará pasar inadvertidos cambios que un buen intérprete hubiera podido poner al descubierto (39). Por otro lado existe el experto ecografista que posee muy buen criterio interpretativo pero no la infor- 
mación clínica suficiente, lo cual puede también conducir a error.

Dado que las posibilidades diagnósticas de los ultrasonidos son muchas, más aún asociándolos al efecto Doppler, se ha pensado en la conveniencia de establecer niveles o grados de idoneidad de quienes hagan uso de esos procedimientos, como también exigir licencia o autorización para ejercer como ecografista profesional (39).

El paso del tiempo ha venido reafirmando la sospecha que se tenía acerca de la inocuidad de los ultrasonidos. Sin embargo, no ha transcurrido todavía el suficiente como para descartar por completo la existencia de riesgos. Por eso hay quienes recomiendan prudencia en su uso, es decir, utilizar la exposición más baja - la estrictamente necesaria- para conseguir la información diagnóstica que se pretende. "El ultrasonido no debe ser un examen frívolo". "La mejor salvaguarda es una buena educación y sólidos conocimientos de los que utilizan el diagnóstico ultrasónico" (52).

\section{Presente de los Ultrasonidos}

Por no ser un ecografista profesional, ni siquiera practicante, me abstengo de analizar a profundidad los avances continuos que la tecnología ha venido introduciendo a los aparatos que se obtienen comercialmente. $\mathrm{Al}$ comparar las máquinas que usó Ian Donald en 1957 (Fig. 2) con las que cuentan en la actualidad los expertos, puede fácilmente deducirse cómo el procedimiento se ha hecho más fácil, práctico y preciso. En 1970 la imagen que se obtenía era "bimodal", estática, en blanco y negro. Diez años más tarde ya era en escala gris, en tiempo real, con alta resolución. Además, sondas o transductores especiales han permitido la exploración intravaginal que hace posible obtener datos más valiosos que los conseguidos a través de la pared abdominal (47). La adición del Doppler capta y registra la onda sanguínea continua y la pulsátil en gris $(6,9,25)$.

Tres son las ayudas que presta en la actualidad la ultrasonografía: diagnóstica, como guía, y pronóstica. Vamos a revisarlas con ánimo de dejar sentado qué puede esperar el obstetra práctico del empleo de los ultrasonidos. Se respalda la revisión con algunas citas bibliográficas recientes y otras claves para quienes quieran profundizar sobre el tema.

\subsection{Como ayuda diagnóstica}

En el primer trimestre del embarazo

Diagnóstica precoz de la gestación. Con un transductor transvaginal de alta frecuencia, con alto poder de resolución, es posible observar el embrión cuando apenas mide 2 ó $3 \mathrm{~mm}$. $(3,46)$.
- Localización ectópica, es decir, cuando cursa fuera de la matriz $(38,35)$.

- Edad gestacional.

- Número de fetos.

- Evolución del embarazo. Precozmente pueden descubrirse las gestaciones frustradas (15).

- Degeneración de las vellosidades coriales (enfermedad trofoblástica gestacional).

En el segundo y tercer trimestres del embarazo

a. En relación con el feto

- Número. No sólo es posible identificar el número de sacos gestacionales, sino también establecer la "corionicidad" placentaria, vale decir, si se trata de un embarazo monocorial o bicorial (13). Igualmente, puede diagnosticarse el llamado síndrome de transfusión gemelo a gemelo (8).

- $\quad$ Edad gestacional (19).

- $\quad$ Ausencia de vitalidad (muerte in útero).

- Desarrollo. Se puede establecer la macrosomía (29), como también el retardo de crecimiento, con la ayuda del efecto Doppler (2).

- Alteraciones morfológicas, mayores y menores (37).

- Sexo. Con una certeza superior al 90 por ciento puede identificarse tempranamente. entre las semanas 16 y 20 (34).

- Situación y presentación.

b. En relación con la placenta

- Sitio de inserción. La placenta previa es registrada fielmente con la sonografía vaginal (17).

- Morfología, maduración y volumen (13, 49, 50).

- Defectos de inserción. No sólo el desprendimiento de áreas reducidas (36), sino también el grado de penetración en el miometrio, como el acretismo (27) y el incretismo (45). 
- Funcionamiento, con la ayuda del efecto Doppler (40).

c. En relación con el líquido amniótico

- Volumen $(10,20)$.

- Infección, mediante el estudio de movimientos corporales y respiratorios del feto (21).

d. En relación con el cordón umbilical

- Ubicación

- Morfología (28, 30).

- Identificación de la vasa previa (28).

4.2 Como guía para procedimientos prenatales invasivos

a. Diagnósticos

- Amniocentesis.

- Cordocentesis $(11,12,41)$.

- $\quad$ Biopsia de vellosidades coriales (7).

b. Terapéuticas (fetoterapia)

- Terapia médica directa, como la administración de digoxina en caso de hydrops (51).

- Transfusión intraperitoneal.

- Exanguinotransfusión (22).

- Nefrostomía en caso de hidronefrosis.

- Drenaje de líquido cefalorraquídeo en caso de hidrocefalia.

- Tratamiento no quirúrgico del embarazo ectópico $(18,48)$.

- Reducción fetal, es decir, aborto selectivo de uno o varios sacos en caso de gestación múltiple $(5,24)$.

c. Pronósticas (porvenir fetal)
- Perfil biofísico fetal cuando se sospecha disfunción placentaria, como sería durante la gestación prolongada (23).

- Maduración y volumen placentario $(49,50)$.

- Volumen del líquido amniótico (10).

- Velocidad del flujo úteroplacentario $(9,25)$.

- Velocidad del flujo umbilical $(6,40)$.

- Velocidad del flujo en aorta descendente y carótida (1).

\section{Panorama Prospectivo}

Con las aportaciones actuales que han quedado reseñadas, pedirle más a la ultrasonografía, ¿no es pedirle mucho? En verdad pudiera pensarse que ya no debe esperarse más. Sin embargo, la tecnología en medicina viene alcanzando posibilidades nunca imaginadas. Por eso las especulaciones lógicas que se hagan no deben recibirse como descabelladas.

Se presagia que con el concurso de las distintas técnicas los resultados serán altamente favorables (26). La suma o combinación de rayos $\mathrm{X}$, de ultrasonidos, de resonancia magnética, y quizás de la tomografía de emisión de positrones, permitirá que se puedan observar en color las imágenes más nítidas, más dinámicas, y con mayor profundidad espacial. Así podrá conocerse mejor la anatomía macroscópica normal y patológica del feto y de la placenta, además de su histología y su fisiología. Nada de raro tiene que la imagenología haga posible la valoración del intercambio de sustancias específicas entre la madre y el feto a través de la placenta, como también la dinámica del líquido amniótico (formación y absorción). El funcionamiento cardiaco, hepático, renal y endocrino del feto podrá, asimismo, ser conocido antes del nacimiento, en virtud de la detección de cambios hemodinámicos, metabólicos y microquími$\cos$.

Cualquier procedimiento invasivo, diagnóstico o terapéutico que en el futuro se proponga irá a ser facilitado por los ultrasonidos, bien como paso previo a ellos o como ayuda guía para que el operador alcance con éxito al blanco que persigue. 
BIBLIOGRAFIA

1. ARDUINI, D.; RIZZO G.; ROMANINI, C. et al. Hemodynamic Changes in growth retarded fetuses during maternal oxygenadministration as predictors of fetal outcome. J Ultrasound Med 8: 193, 1989.

2. BENSON, C.B.; DOUBILET, P.M. Doppler criteria for intrauterine growth retardation: Predictive values. J. Ultrasound Med 7: 655, 1988.

3. BERNASCHEK, G.; RUDELSTORFER, R.; CSAICSICH P. Vaginal sonography versus serum human chorionic gonadotropin in early detection of pregnancy. Am J Obstet Gynec 158: 608, 1988.

4. BLOCH, F. Nuclear Induction. Phys Rev 69: 127, 1946.

5. BRANDES, J.M.; ITSKOVITZ, J.; TIMOR-TRITSCH I.F. et al. Reduction of the number of embryos in a multiple pregnancy: quintuplet to triplet. Fertil Steril 48: 326, 1987.

6. BRAR, H.S.; MEDEARIS, A.L.; DeVORE, G.R.; PLATT L.D. A comparative study of fetal umbilical velocimetry with continous and pulled wave Doppler ultrasonography in high-risk pregnancies: Relationship to outcome. Am J Obstet Gynec 160: 375, 1989.

7. BRAMBATI, B.; OLDRINI, A.; LANZANI, A. Transabdominal chorionic villus sampling: $A$ freehand ultrasound-guided technique. Am J Obstet Gynec 157: 134, 1987.

8. BROWN, D.L. Twin-twin transfusion syndrome: Sonographic findings. Radiology 170: 61, 1989.

9. CAMPBELL, S.; DIAZ, R.J.; GRIFFIN, D.R. et al. New Doppler Technique for assessing uteroplacental blodd flow. Lancet 1: 875,1983

10. CHAMBERLAIN, P.F.; MANNING, F.A.; MORRISON, I. et al. The relationship of marginal and decreased amniotic fluid volumes to perinatal outcome. Am. J. Obst. Gynec. 150: 245, 1984.

11. DAFFOS, F.; CAPELLA, M.; FORESTIER, F. A. new procedure for fetal blood sampling in utero: Preliminary results of fifty-three cases. Am. J. Obstet. Gynec. 146, 985, 1983.

12. DAFFOS, F.; CAPELLA, M.; FORESTIER, F. Fetal blood sampling during pregnancy with use of a needle guided by ultrasound: a study of 606 consecutive cases. Am. J. Obstet. Gynec. 153: 655, 1985

13. D'ALTON, M.E.; DUDLEY, D.K. The ultrasonographic prediction of chorionicity in twin gestation. Am. J. Obstet. Gynec. 160: 557, 1989.

14. DARNEY, P.D.; SWEET, R.L. Routine intraoperative ultrasonography for second trimester abortion reduces incidence of uterine perforation. J. Ultrasound Med 8: 71, 1989.

15. DE CRESPIGNY, L. Early diagnosis of pregnancy failure with transvaginal ultrasound. Am. J. Obstet. Gynec. 159: 408, 1988.

16. EASTMAN KODAK COMPANY. Medical Diagnostic Ultrasound: A Retrospective on its 40th Anniversary. Printed in USA, 1988.
17. FARINE, D.; FOX, H.E.; JACOBSON, S. et al. Vaginal ultrasound for diagnosis of placenta previa. Am. J. Obstet. Gynec. 159: 566, 1988.

18. FEICHTINGER, W.; KEMETER, P. Conservative treatment of ectopic pregnancy by transvaginal aspiration under sonographic control and methotrexate injection. Lancet 1: 381, 1987.

19. GOLDENBERG, R.L.; DAVIS, R.O.; CUTTER, G.R. et al. Prematurity postdates, and growth retardation: The influence of use of ultrasonography on reported gestational age. Am. J. Obstet. Gynec. 160: 462, 1989.

20. GOLDSTEIN, R.B.; FILLY, R,A. Sonographic estimation of amniotic fluid volume. J. Ultrasound Med. 7: 363, 1988.

21. GOLDSTEIN, I.; ROMERO, R.; MERRILL, S. et al. Fetal body and breathing movements as predictors of intraamniotic infection in preterm premature rupture of membranes. Am. J. Obstet. Gynec. 159: 363, 1988.

22. GRANNUM, P.A.; COPEL, J.A.; PLAXE, S.C. et al. In utero exchange transfusion by direct intravascular injection in severe erythroblastosis fetalis. N. Engl. J. Med. 154: 1105, 1986.

23. HANN, L.; Mc. ARDLE, C.; SACHS, B. Sonographic biophysical profile in the postdate pregnancy. J. Ultrasound Med. 6: $191,1987$.

24. ITSKOVITZ, J.; BOLDES, R.; THALER, I. et al. Transvaginal ultrasonography-guided aspiration of gestational sacs for selective abortion in multiple pregnancy. Am. J. Obstet. Gynec. 160: 215, 1989.

25. McCALLUM, W.D.; WILLIAMS, C.S.; NAPEL, S.; DAIGLE, R.E. Fetal blood velocity waveforms. Am. J. Obstet. Gynec. 132: 425, 1978.

26. MEDINA, L.S.; MEDINA, J. La radiología una revolución. Revista CES Medicina 3: 53, 1989.

27. MENDUNCA, L.K. Sonographic diagnosis of placenta accreta: Presentation of six cases. J. Ultrasound Med. 7: 211, 1988.

28. MESSER, R.H.; GOMEZ, A.R.; YAMBOTJ. Antepartum testing for vasa previa: Current standard of care. Am. J. Obstet. Gynec. 156: 1459, 1987.

29. MILLER, J.M.; BROWN, H.L.; KHANLI, O.F. et al. Ultrasonographic identification of the macrosomic fetus. Am. J. Obstet. Gynec. 159: 1110, 1988.

30. POLLACK, M.S.; BOUND, L.M. Hemangioma of the umbilical cord. Sonographic appearance. J. Ultrasound Med. 8: $163,1989$.

31. POWELL, M.C.; BUCKLEY, J.; WORTHINGTON, B. Magnetic Resonance Imaging and hydatidiform mole. Br. J. Radiol. 59: 561, 1986.

32. PURCELL, E. Resonance absorption by nuclear magnetic moments in a solid. Phys Rev. 69: 37, 1946.

33. QUIJANO, A. Resonancia Nuclear Magnética. Neurología Col. 8: 111, 1984. 
34. REECE, E.A.; WINN, H.N.; WAN, M. et al. Can ultrasonography replace amniocentesis in fetal gender determination during the early second trimester? Am. J. Obstet. Gynec. 156: 579, 1987.

35. REMPEN, A. Vaginal sonography in ectopic pregnancy. J. Ultrasound Med. 7: 381, 1988.

36. RIVERA-ALSINA, M.; SALDANA, L.R.; MAKLAN, N.: KOPS, $S$. The use of ultrasound in the expectant management of abruptio placentae. Am. J. Obstet. Gynec. 146: 924, 1983.

37. ROMERO, R.; OYARZUN, E.; SIRTORI, M.; HOBBINS, J.C. Detection and management of anatomic congenital anomalies. A new obstetric challenge. Obstet Gynecol Clin North Am 15: 215, 1988.

38. ROMERO, R.; KADAR, N.; CASTRO, D. et al. The value of adnexal sonographic findings in the diagnosis of ectopic pregnancy. Am. J. Obstet. Gynec. 158: 52, 1988.

39. SACK, R.A.; MAHARRY, J.H. Misdiagnosis in obstetric and gynecologic ultrasound examination: Causes and possible solutions. Am. J. Obstet. Gynec. 158: 1260, 1988.

40. SCHULMAN, H. The clinical implications of Doppler ultrasound analysis of the uterine and umbilical arteries. Am. J. Obstet. Gynec. 156: 887, 1987.

41. SHAH, D.M.; JEANTY, P.; DEU, V.G. et al. Diagnosis of trisomy 18 in monozygotic twins by cordocentesis. Am. J. Obstet. Gynec. 160: 214, 1989.

42. SHOR-PINSKER, V.; PAEZ-JIMENEZ, F.J. Resonancia Magnética en Perinatología. Perinatal Reprod. Hum. 3: 31, 1989.

43. SMITH, F.W.; ADAM, A.H. NRM. Imaging in pregnancy. Lancet $1(8)$ : $61,1983$.
44. SMITH, F.W.; KENT, C.; ABAMOVICH, D. Nuclear Magnetic Resonance Imaging. A new look at the fetus. Br. J. Obstet. Gynecol. 92: 1024, 1985.

45. TABSH, K.M.A.; BRINKMAN, C.R.; KING, W. Ultrasound diagnosis of placenta increta. J. Clin. Ultrasound 10:288, 1982.

46. TIMOR-TRITSCH, I.E.; FARINE, D.; ROSEN, M.G.; A close look at early embryogenic development with the high-frequency transvaginal transducer. Am. J. Obstet. Gynec. 159: 676, 1988.

47. TIMOR-TRITSCH, I.E.; BAR-YAM, Y.; ELGALI, S.; ROTTEM, $\mathrm{S}$. The technique of transvaginal sonography with the use of a $6.5 \mathrm{MHz}$ probe. Am. J. Obstet. Gynec. 158: 1019, 1988.

48. TIMOR-TRITSCH, I.; BAXI, L.; PEISNER, D.B. Transvaginal salpingocentesis: A new technique for treating ectopic pregnancy. Am. J. Obstet. Gynec. 160: 459, 1989.

49. WOLF, H.; OOSTIN, H.; TREFFERS, P.E. Placental volume measurement by ultrasonography. Evaluation of the method. Am. J. Obstet. Gynec. 156: 1191, 1987.

50. WOLF, H.; OOSTIN, H.; TREFFERS, P.E. Second-trimester placental volume measurement by ultrasound: Prediction of fetal outcome. Am. J. Obstet. Gynec. 160: 121, 1989.

51. YOUNIS, J.S.; GRANAT, M. Insufficient transplacental digoxin transfer in severe hydrops fetalis. Am. J. Obstet. Gynec. 157: 1268, 1987.

52. ZISKIN, M.C. The prudent use of diagnostic ultrasound (Editorial). J. Ultrasound. Med. 6: 415, 1987. 